\title{
A Systemic Lupus Erythematosus Patient With Febrile Neutropenia Developing After Intraocular Administration of Bevacizumab
}

\author{
Hamdi SÖZEN, ${ }^{1}$ Gönen MENGİ, ${ }^{2}$ Bülent HÜDDAM, ${ }^{3}$ Selmin ÇAYLAK, ${ }^{1}$ \\ Mehmet DEVEER, ${ }^{4}$ Volkan KARAKUŞ, ${ }^{5}$ Burak Ekrem ÇİTIL ${ }^{6}$ \\ ${ }^{1}$ Department of Infectious Disease, Medical Faculty of Muğla Sitkı Koçman University, Muğla, Turkey \\ ${ }^{2}$ Department of Rheumatology, Medical Faculty of Muğla Sıtkı Koçman University, Muğla, Turkey \\ ${ }^{3}$ Department of Nephrology, Medical Faculty of Muğla Sttkı Koçman University, Muğla, Turkey \\ ${ }^{4}$ Department of Radiology, Medical Faculty of Muğla Sıtkı Koçman University, Muğla, Turkey \\ ${ }^{5}$ Department of Hematology, Medical Faculty of Muğla Sıtkı Koçman University, Muğla, Turkey \\ ${ }^{6}$ Department of Microbiology, Medical Faculty of Muğla Sıtkı Koçman University, Muğla, Turkey
}

\begin{abstract}
In this article, we report a case with febrile neutropenia occurring after implementation of intraocular bevacizumab. A 60-year-old female patient who had received intravitreal bevacizumab applied to hospital for oral lesions accompanying fever and neutropenia. In spite of empiric antibiotherapy, patient's clinical condition did not improve. Her sputum culture yielded Stenotrophomonas maltophilia. Trimethoprim/ sulfamethoxazole and levofloxacin were commenced according to antibiogram. In immunologic evaluation, antinuclear antibodies and anti-double stranded deoxyribonucleic acid were positive as compatible with systemic lupus erythematosus. After proper antibiotic treatment, patient's clinical symptoms resolved. Development of severe neutropenia after procedures which are not expected to create systemic adverse effects should bring to mind underlying immunosuppressive diseases such as systemic lupus erythematosus.

Keywords: Bevacizumab; febrile neutropenia; Stenotrophomonas maltophilia; systemic lupus erythematosus.
\end{abstract}

Systemic lupus erythematosus (SLE) is a prototypic systemic autoimmune disease with variable multisystemic involvement and heterogeneous clinical features, ranging from mild to life threatening. ${ }^{1}$ Although hematological abnormalities are commonly found in SLE, severe polymorphonuclear leukocyte depletion is rare. Severe leukopenia seen in SLE is usually associated with drug toxicity. ${ }^{2}$

Bevacizumab is a recombinant humanized monoclonal antibody directed against vascular endothelial growth factor and it is used worldwide in ophthalmological conditions such as age related macular degeneration. ${ }^{3}$ Leukopenia is one of the most important side effects of bevacizumab, but as far as we know so far, it has not been reported after intravitreal bevacizumab. ${ }^{4}$

In this article, we report a case with febrile neutropenia occurring after the implementation of intravitreal bevacizumab in a patient diagnosed as SLE after the intervention.

\section{CASE REPORT}

A 60-year-old female patient with no chronic disease except epilepsy which has been under control with oxcarbazepine and levetiracetam for 20 years applied to hospital. She received intravitreal bevacizumab injection 
(1.25 mg/0.05 mL) for age related macular degeneration at a private clinic and four days after this treatment, painful lesions emerged in her mouth accompanied with fever. She was admitted to the internal medicine clinic with these complaints and took diagnosis of febrile neutropenia. In spite of meropenem and amikacin treatment for four days, fever and neutropenia did not resolve; so, the patient was referred to our unit. On physical examination, her body temperature was $39.4{ }^{\circ} \mathrm{C}$, multiple ulcers and white plaque lesions were observed in her mouth, and rales were present in the left lower lung zone compatible with pneumonia. X-ray revealed consolidation area with irregular borders at the middle zone of the left lung (Figure 1). The laboratory findings were as follows: white blood cells (WBC) $0.7 \mathrm{~K} / \mu \mathrm{l}$, neutrophils (absolute neutrophil count; ANC) $0.05 \mathrm{~K} / \mu \mathrm{l}$, platelets (PLT) $411 \mathrm{~K} / \mu \mathrm{l}$, hemoglobin $(\mathrm{Hgb}) 10.3 \mathrm{~g} / \mathrm{dL}$, C-reactive protein (CRP) 348.9 (0-5) $\mathrm{mg} / \mathrm{L}$, erythrocyte sedimentation rate (ESR) $101 \mathrm{~mm} /$ hour, procalcitonin $3.4 \mu \mathrm{g} / \mathrm{mL} \quad(<0.05 \mu \mathrm{g} / \mathrm{mL})$, total protein $5.9 \mathrm{mg} / \mathrm{dL}$, and albumin $2.5 \mathrm{mg} / \mathrm{dL}$. After obtaining the routine cultures, meropenem ( $3 \times 1 \mathrm{~g}$ intravenous), vancomycin $(2 \times 1 \mathrm{~g}$ intravenous), liposomal amphotericin B (150 mg intravenous), and filgrastim (granulocyte colonystimulating factor analog, $30 \mathrm{MU}$ subcutaneous) were commenced. Thorax computed tomography showed pleural effusion accompanied by basal consolidation area and fibrous bands in the left lung compatible with pneumonia (Figure 2). On the fourth day of the treatment, patient's fever and other clinical symptoms had not improved

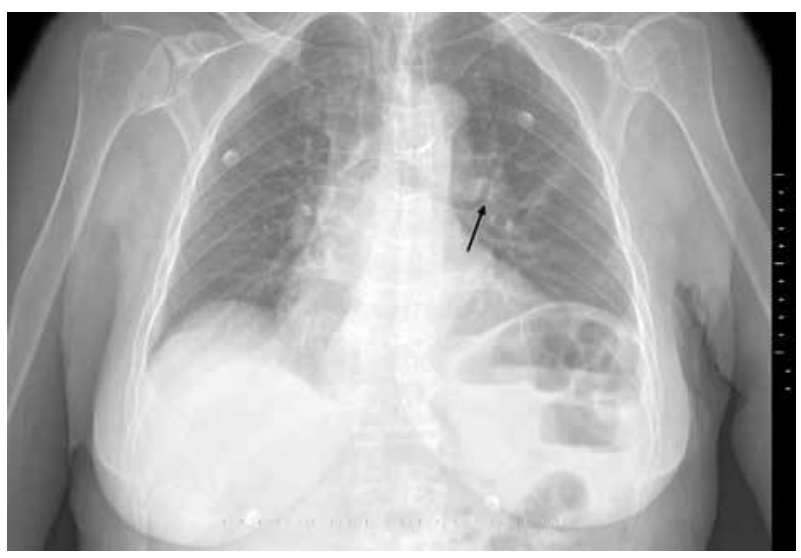

Figure 1. Consolidation area accompanying linear band at left mid-lower zone of lung. and laboratory results were as follows: WBC $1 \mathrm{~K} / \mu \mathrm{L}$, ANC $0.1 \mathrm{~K} / \mu \mathrm{L}$, Hgb $8.4 \mathrm{~g} / \mathrm{dL}$, PLT $535 \mathrm{~K} / \mu \mathrm{L}$, CRP $144 \mathrm{mg} / \mathrm{L}$, ESR $140 \mathrm{~mm}$ /hour, and procalcitonin $2.24 \mu \mathrm{g} / \mathrm{mL}$. Patient's sputum culture yielded Stenotrophomonas maltophilia susceptible to trimethoprim/sulfamethoxazole and levofloxacin. Previous antibiotics were discontinued and trimethoprim/sulfamethoxazole and levofloxacin were started. Besides antibiotic therapy, anti-epileptic regime was maintained. The infectious agents which can cause neutropenia were also excluded. Antinuclear antibodies were detected as positive $(++)$ by immunofluorescence assay at 1/100 titration and homogenous pattern. Also, anti-double stranded deoxyribonucleic acid was positive $(+++)$ by immunoblotting techniques. In urinalysis, proteinuria was detected. Following the detection of protein in urinalysis, protein in 24 hour urine was measured as $735 \mathrm{mg}$. At the fifth day of the treatment with trimethoprim/ sulfamethoxazole and levofloxacin, patient's body temperature returned to normal and clinical symptoms improved. Laboratory results were as follows: WBC $7.9 \mathrm{~K} / \mu \mathrm{L}$, ANC $4.5 \mathrm{~K} / \mu \mathrm{L}$, Hgb $8.5 \mathrm{~g} / \mathrm{dL}$, PLT $205 \mathrm{~K} / \mu \mathrm{L}$, CRP 10.5 (0-5) mg/L, ESR $36 \mathrm{~mm} / \mathrm{h}$, procalcitonin $0.08 \mu \mathrm{g} / \mathrm{mL}$, blood urea nitrogen $27 \mathrm{mg} / \mathrm{dL}$, and creatinine $0.8 \mathrm{mg} / \mathrm{dL}$. An evaluation of kidney biopsy performed due to significant proteinuria revealed mild staining with immunoglobulins and complements. Kidney biopsy was found compatible with class III + class $\mathrm{V}$ lupus nephritis.

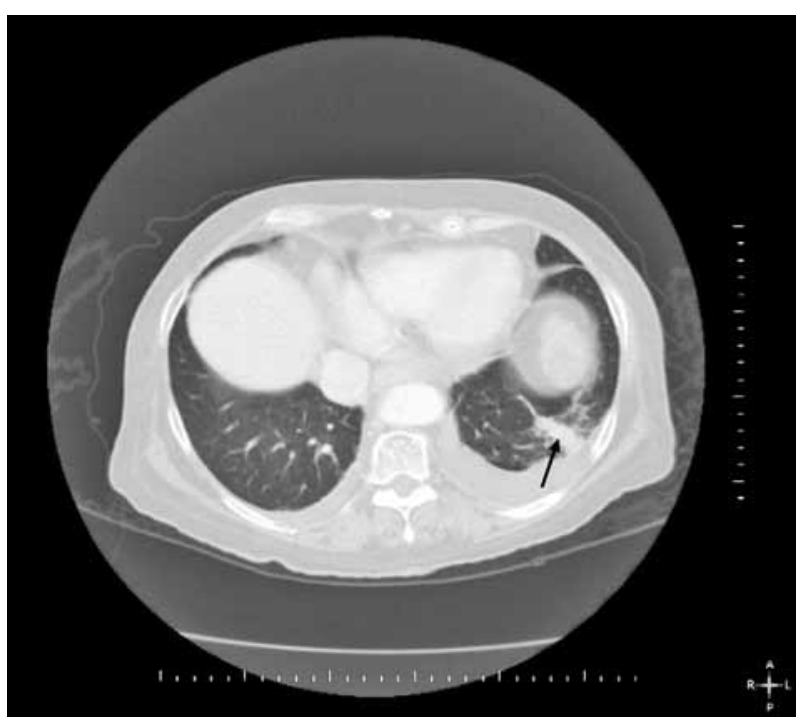

Figure 2. Consolidation area and fibrous septa at posterobasal segment of left lower lobe lung zone. 
Mycophenolate mofetil and prednisolone were administered for the management of SLE complicated with nephritis by the consensus of rheumatologists and nephrologists. Hydroxychloroquine could not be used because of maculopathy of the patient. In the followup period, the laboratory findings improved as follows: WBC $5.6 \mathrm{~K} / \mu \mathrm{L}$, ANC $4.6 \mathrm{~K} / \mu \mathrm{L}$, Hgb $10.4 \mathrm{~g} / \mathrm{dL}$, PLT $319 \mathrm{~K} / \mu \mathrm{L}, \mathrm{CRP} 3.8$ (0-5) mg)/L, ESR $18 \mathrm{~mm} /$ hour, normal complement levels, creatinine $0.7 \mathrm{mg} / \mathrm{dL}$, blood urea nitrogen $16 \mathrm{mg} / \mathrm{dL}$, and $516 \mathrm{mg} / 24$ hour urine protein. A written informed consent was obtained from the patient.

\section{DISCUSSION}

Systemic lupus erythematosus is a complex rheumatic disease with diverse clinical manifestations. ${ }^{5}$ Typical symptoms and findings are found in a minority of patients. If clinical symptoms are unclear, diagnosis may be delayed. Hematological complications are usually seen in SLE. Although leukopenia is detected in approximately $50 \%$ of SLE patients, only $17 \%$ of their WBC is lower than $1.0 \mathrm{~K} / \mu \mathrm{L}$. A small percentage of patients develop life-threatening neutropenia which may lead to opportunistic infection. ${ }^{1}$ In SLE, leukopenia may result from bone marrow failure or excessive peripheral cell destruction. Adverse effects of drugs and infections are other common reasons. ${ }^{6}$ Infection is a frequent problem in patients with SLE, particularly in hospitalized patients due to complications of the disease. ${ }^{7}$ Our patient was also examined by a hematologist. Hematological pathologies which may cause neutropenia were excluded by normal bone marrow biopsy. Also, infectious factors were ostracized by serological tests.

Until bevacizumab injection, our patient did not have neutropenia or associated infectious disease history. Although systemic administration of bevacizumab may result in fatal side effects such as neutropenia and bleeding, the frequency of intravitreal injection's side effects is low. ${ }^{4}$ Intravitreal bevacizumab might cause some systemic side effects such as stroke and hypertension; however, to our knowledge, severe neutropenia has not been reported yet. ${ }^{8}$ Although systemic complications are rarely observed in patients exposed to intravitreal bevacizumab, the presence of SLE may be trigger severe neutropenia. Neutropenia is one of the most important risk factors associated with Stenotrophomonas maltophilia infection. ${ }^{9}$ Particularly in hospitalized patients, Stenotrophomonas maltophilia is an opportunistic pathogen with multiple drug resistance..$^{10}$ Treatment may be challenging due to its intrinsic resistance to multiple antibiotics.

In conclusion, underlying immunosuppressive diseases such as SLE should be considered when neutropenia and severe systemic infections develop owing to locally invasive procedures which are not expected to create systemic side effects. Furthermore, intravitreal bevacizumab should be applied more cautiously in patients previously diagnosed as SLE.

\section{Declaration of conflicting interests}

The authors declared no conflicts of interest with respect to the authorship and/or publication of this article.

\section{Funding}

The authors received no financial support for the research and/or authorship of this article.

\section{REFERENCES}

1. Fayyaz A, Igoe A, Kurien BT, Danda D, James JA, Stafford HA, et al. Haematological manifestations of lupus. Lupus Sci Med 2015;2:000078.

2. Sakai T, Hatano Y, Abe I, Ishii K, Fujiwara S. A case of an SLE patient with febrile neutropenia who experienced exacerbation of cutaneous manifestations after the administration of G-CSF. Mod Rheumatol 2013;23:1231-6.

3. Popescu V, Pricopie S, Totir M, lancu R, Yasyn S, Alexandrescu C. Clinical use of Bevacizumab in treating refractory glaucoma. J Med Life 2015;8:8-12.

4. Ranpura V, Hapani S, Wu S. Treatment-related mortality with bevacizumab in cancer patients: a meta-analysis. JAMA 2011;305:487-94.

5. Pons-Estel GJ, Alarcón GS, Scofield L, Reinlib L, Cooper GS. Understanding the epidemiology and progression of systemic lupus erythematosus. Semin Arthritis Rheum 2010;39:257-68.

6. Hepburn AL, Narat S, Mason JC. The management of peripheral blood cytopenias in systemic lupus erythematosus. Rheumatology (Oxford) 2010;49:2243-54.

7. Onur ND, Duzgun N, Olmez U, Ates A. Miliary tuberculosis in a patient with systemic lupus erithematosus with abscess formation in the upper extremities. J Ankara Med Sch 2003;25:35-40. 
8. Wang W, Zhang X. Systemic adverse events after intravitreal bevacizumab versus ranibizumab for agerelated macular degeneration: a meta-analysis. PLoS One 2014;9:109744.

9. Falagas ME, Kastoris AC, Vouloumanou EK, Rafailidis PI, Kapaskelis AM, Dimopoulos G. Attributable mortality of Stenotrophomonas maltophilia infections: a systematic review of the literature. Future Microbiol 2009;4:1103-9.

10. Lai $\mathrm{CH}$, Chi CY, Chen HP, Chen TL, Lai CJ, Fung $\mathrm{CP}$, et al. Clinical characteristics and prognostic factors of patients with Stenotrophomonas maltophilia bacteremia. J Microbiol Immunol Infect 2004;37:350-8. 